Article

\title{
Extremal Trees with Respect to the Difference between Atom-Bond Connectivity Index and Randić Index
}

\author{
Wan Nor Nabila Nadia Wan Zuki ${ }^{1}$, Zhibin Du ${ }^{2,3}{ }^{\circledR}$, Muhammad Kamran Jamil ${ }^{4}$ \\ and Roslan Hasni ${ }^{1 \text {,* }}$ \\ 1 Faculty of Ocean Engineering Technology and Informatics, Universiti Malaysia Terengganu, \\ Kuala Nerus 21030, Terengganu, Malaysia; nadiazuki96.nz@gmail.com \\ 2 School of Software, South China Normal University, Foshan 528225, China; duzhibin@zqu.edu.cn \\ 3 School of Mathematics and Statistics, Zhaoqing University, Zhaoqing 526061, China \\ 4 Department of Mathematics, Riphah Institute of Computing and Applied Sciences, Riphah International \\ University, Lahore 46000, Pakistan; kamran.jamil@riphah.edu.pk \\ * Correspondence: hroslan@umt.edu.my
}

Received: 23 July 2020; Accepted: 23 August 2020; Published: 25 September 2020

\begin{abstract}
Let $G$ be a simple, connected and undirected graph. The atom-bond connectivity index $(A B C(G))$ and Randić index $(R(G))$ are the two most well known topological indices. Recently, Ali and $\mathrm{Du}$ (2017) introduced the difference between atom-bond connectivity and Randić indices, denoted as $A B C-R$ index. In this paper, we determine the fourth, the fifth and the sixth maximum chemical trees values of $A B C-R$ for chemical trees, and characterize the corresponding extremal graphs. We also obtain an upper bound for $A B C-R$ index of such trees with given number of pendant vertices. The role of symmetry has great importance in different areas of graph theory especially in chemical graph theory.
\end{abstract}

Keywords: Randić index; Atom-bond connectivity index; tree

\section{Introduction}

Let $G$ be a simple, connected and undirected graph. having $V(G)$ and $E(G)$ as the set of vertices and edges respectively. The number of vertices and edges in $G$ are denoted by $n m$, respectively. Let $d_{u}$ denotes the degree of vertex $u$ in $G$, while $\triangle(G)$ and $\delta(G)$ are used to denote the maximum and minimum degree of $G$. The distance $d_{G}(x, y)$ between vertices $x$ and $y$ is defined as the length of any shortest path in $G$ connecting $x$ and $y$. The eccentricity of $v_{i}$ in $G$ is defined as $e_{i}=\max _{v_{j} \in V(G)} d_{G}\left(v_{i}, v_{j}\right)$. For more concepts and terminologies in Graph Theory, we refer to [1].

Topological indices is one of the useful tools of graph theory [2]. Molecular compounds are often modeled by molecular graphs are used to represent the molecules and molecular compounds with the help of lines and dots. In study of QSPR/QSAR, topological indices are considered as one of the useful topics [3].

In 1975, Randić [4] defined the Randić index as follows:

$$
R(G)=\sum_{u v \in E(G)} \frac{1}{\sqrt{d_{u} d_{v}}}
$$

Details about Randić index and most of its mathematical properties can be found in [5-10]. 
Estrada et al. [11] proposed the atom-bond connectivity ( $A B C$ for short) for a molecular graph as

$$
A B C(G)=\sum_{u v \in E(G)} \sqrt{\frac{d_{u}+d_{v}-2}{d_{u} d_{v}}} .
$$

This index became popular only ten years later, when the paper [12] was published. For the details, see the surveys [13], the recent papers [14-19] and the references cited therein.

Nowadays, studying the relationship or comparison between topological indices, see [20-23], is becoming popular. Recently, Ali and Du [24] investigated extremal binary and chemical trees results for the difference between $A B C$ and $R$ indices. A tree with maximum degree at most three or four called a binary and chemical tree, respectively.

For a connected graph $G$ of order at least 3, the difference between $A B C$ and $R$ is represented as (see [24])

$$
(A B C-R)(G)=\sum_{u v \in E(G)} \frac{\sqrt{d_{u}+d_{v}-2}-1}{\sqrt{d_{u} d_{v}}} .
$$

Note that $(A B C-R)(G) \geq 0$ and equality holds if and only if $G=P_{3}$. So in our discussion we consider $n \geq 4$.

In this paper, motivated by the results in [24], we further investigated the extremal chemical trees for $A B C-R$. Moreover, maximal trees with fixed number of pendant vertices are also investigated for $A B C-R$ index. The techniques used in this paper are very similar to that of Refs. [19,24,25].

\section{Preliminary Results}

Let the number of edges connecting the vertices of degree $p$ and $q$ is denoted by $x_{p, q}$. In term of $p, q$ and $x_{p, q} A B C-R$ can be rewritten as follows [24]:

$$
(A B C-R)(G)=\sum_{\delta \leq p \leq q \leq \Delta} \frac{\sqrt{p+q-2}-1}{\sqrt{p q}} x_{p, q} .
$$

Let $n_{p}$ be the number of vertices of degree $p$ in $G$, where $1 \leq p \leq 4$. Then for any $n$-vertex chemical tree the following system of equations holds (see $[19,24])$ :

$$
\begin{gathered}
n_{1}+n_{2}+n_{3}+n_{4}=n, \\
n_{1}+2 n_{2}+3 n_{3}+4 n_{4}=2(n-1), \\
x_{1,2}+x_{1,3}+x_{1,4}=n_{1}, \\
x_{1,2}+2 x_{2,2}+x_{2,3}+x_{2,4}=2 n_{2}, \\
x_{1,3}+x_{2,3}+2 x_{3,3}+x_{3,4}=3 n_{3} \\
x_{1,4}+x_{2,4}+x_{3,4}+2 x_{4,4}=4 n_{4} .
\end{gathered}
$$

From Equations (2) and (3), it follows that

$$
n_{2}+2 n_{3}+3 n_{4}=n-2,
$$

and thus,

$$
n \equiv n_{2}+2 n_{3}+2(\bmod 3) .
$$

By solving the sysmtem of Equations (2)-(7), the values of $x_{1,4}$ and $x_{4,4}$ are, respectively, given as below (see also Refs. [24,26]): 


$$
\begin{gathered}
x_{1,4}=\frac{2 n+2}{3}-\frac{4}{3} x_{1,2}-\frac{10}{9} x_{1,3}-\frac{2}{3} x_{2,2}-\frac{4}{9} x_{2,3}-\frac{1}{3} x_{2,4}-\frac{2}{9} x_{3,3}-\frac{1}{9} x_{3,4} \\
x_{4,4}=\frac{n-5}{3}+\frac{1}{3} x_{1,2}+\frac{1}{9} x_{1,3}-\frac{1}{3} x_{2,2}-\frac{5}{9} x_{2,3}-\frac{2}{3} x_{2,4}-\frac{7}{9} x_{3,3}-\frac{8}{9} x_{3,4}
\end{gathered}
$$

Note that the detailed calculation of obtaining the values for $x_{1,4}$ and $x_{4,4}$ can be referred in [26]. By substituting these values of $x_{1,4}$ and $x_{4,4}$ in Equation (1), one has:

$$
\begin{aligned}
(A B C-R)(G)= & \frac{4 \sqrt{3}+\sqrt{6}-5}{12} n+\frac{4 \sqrt{3}-5 \sqrt{6}+1}{12}-\frac{8 \sqrt{3}-\sqrt{6}-7}{12} x_{1,2} \\
& -\frac{32 \sqrt{3}-13 \sqrt{6}-19}{36} x_{1,3}-\frac{4 \sqrt{3}+\sqrt{6}-6 \sqrt{2}+1}{12} x_{2,2} \\
& -\frac{8 \sqrt{3}+11 \sqrt{6}-18 \sqrt{2}-13}{36} x_{2,3}-\frac{2 \sqrt{3}+2 \sqrt{6}-3 \sqrt{2}-4}{12} x_{2,4} \\
& -\frac{4 \sqrt{3}+7 \sqrt{6}-23}{36} x_{3,3}-\frac{4 \sqrt{3}+4 \sqrt{6}-3 \sqrt{15}-5}{18} x_{3,4} .
\end{aligned}
$$

Let

$$
\begin{aligned}
\theta= & \frac{8 \sqrt{3}-\sqrt{6}-7}{12} x_{1,2}+\frac{32 \sqrt{3}-13 \sqrt{6}-19}{36} x_{1,3}+\frac{4 \sqrt{3}+\sqrt{6}-6 \sqrt{2}+1}{12} x_{2,2} \\
& +\frac{8 \sqrt{3}+11 \sqrt{6}-18 \sqrt{2}-13}{36} x_{2,3}+\frac{2 \sqrt{3}+2 \sqrt{6}-3 \sqrt{2}-4}{12} x_{2,4} \\
& +\frac{4 \sqrt{3}+7 \sqrt{6}-23}{36} x_{3,3}+\frac{4 \sqrt{3}+4 \sqrt{6}-3 \sqrt{15}-5}{18} x_{3,4} .
\end{aligned}
$$

Then Equation (9) can be rewritten as

$$
(A B C-R)(G)=\frac{4 \sqrt{3}+\sqrt{6}-5}{12} n+\frac{4 \sqrt{3}-5 \sqrt{6}+1}{12}-\theta .
$$

since

$$
\begin{aligned}
\theta \approx & 0.367243 x_{1,2}+0.127285 x_{1,3}+0.157701 x_{2,2}+0.0651375 x_{2,3} \\
& +0.0100367 x_{2,4}+0.0298509 x_{3,3}+0.00595623 x_{3,4} .
\end{aligned}
$$

From Equation (12) we have $\theta \geq 0$. Moreover Equation (11) implies that a chemical tree which gives the minimum value of $\theta$ will produce the maximum of $(A B C-R)$.

Theorem 1 ([24]). Consider the set of all n-vertex chemical trees.

(1) Suppose that $n \equiv 0(\bmod 3)$.

(1.1) For $n \geq 9$, the maximum $A B C-R$ value is

$$
\frac{4 \sqrt{3}+\sqrt{6}-5}{12} n+\frac{3+2 \sqrt{2}-3 \sqrt{6}}{4}
$$

which is uniquely attained by those trees that contain a unique vertex of degree 2 and no vertex of degree 3 , that is, $n_{2}=1$ and $n_{3}=0$, such that the unique vertex of degree 2 is adjacent to two vertices of degree 4 , that is, $x_{1,2}=0$ and $x_{2,4}=2$. 
(1.2) For $n \geq 21$, the second maximum $A B C-R$ value is

$$
\frac{4 \sqrt{3}+\sqrt{6}-5}{12} n+\frac{4 \sqrt{15}-7 \sqrt{6}-4 \sqrt{3}+7}{4},
$$

which is uniquely attained by those trees that contain no vertex of degree 2 and exactly two vertices of degree 3 , that is, $n_{2}=0$ and $n_{3}=2$, such that each vertex of degree 3 is adjacent to three vertices of degree 4 , that is, $x_{1,3}=x_{3,3}=0$ and $x_{3,4}=6$.

(1.3) For $n \geq 21$, the third maximum $A B C-R$ value is

$$
\frac{4 \sqrt{3}+\sqrt{6}-5}{12} n+\frac{4 \sqrt{15}-4 \sqrt{3}-9 \sqrt{6}+11}{6}
$$

which is uniquely attained by those trees that contain no vertex of degree 2 and exactly two vertices of degree 3 , which are adjacent, that is, $n_{2}=0, n_{3}=2$, and $x_{3,3}=1$ such that each vertex of degree 3 is adjacent to exactly two vertices of degree 4 , that is, $x_{1,3}=0$ and $x_{3,4}=4$.

(2) Suppose that $n \equiv 1(\bmod 3)$.

(2.1) For $n \geq 13$, the maximum $A B C-R$ value is

$$
\frac{4 \sqrt{3}+\sqrt{6}-5}{12} n+\frac{11+6 \sqrt{15}-4 \sqrt{3}-13 \sqrt{6}}{12}
$$

and the equality holds if and only if $n_{2}=0$ and $n_{3}=1$ such that $x_{1,3}=0$ and $x_{3,4}=3$.

(2.2) For $n \geq 13$, the second maximum $A B C-R$ value is

$$
\frac{4 \sqrt{3}+\sqrt{6}-5}{12} n+\frac{12 \sqrt{2}-13 \sqrt{6}-4 \sqrt{3}+17}{12},
$$

which is uniquely attained by those trees that contain exactly two vertices of degree 2 and no vertex of degree 3 , that is, $n_{2}=2$ and $n_{3}=0$, such that either vertex of degree 2 is adjacent to two vertices of degree 4 , that is, $x_{1,2}=x_{2,2}=0$ and $x_{2,4}=4$.

(2.3) For $n \geq 25$, the third maximum $A B C-R$ value is

$$
\frac{4 \sqrt{3}+\sqrt{6}-5}{12} n+\frac{12 \sqrt{15}-6 \sqrt{2}-25 \sqrt{6}-16 \sqrt{3}+29}{12}
$$

which is uniquely attained by those trees that contain a unique vertex of degree 2 and exactly two vertices of degree 3 , that is, $n_{2}=1$ and $n_{3}=2$, such that each vertex of degree 2 and 3 is adjacent to only vertices of degree 4 , that is, $x_{1,2}=x_{1,3}=x_{2,3}=x_{3,3}=0, x_{2,4}=2$, and $x_{3,4}=6$.

(3) Suppose that $n \equiv 2(\bmod 3)$.

(3.1) For $n \geq 5$, the maximum $A B C-R$ value is

$$
\frac{4 \sqrt{3}+\sqrt{6}-5}{12} n+\frac{4 \sqrt{3}-5 \sqrt{6}+1}{12}
$$

which is uniquely attained by those trees that contain no vertex of degree 2 or 3 , that is, $n_{2}=$ $n_{3}=0$.

(3.2) For $n \geq 17$, the second maximum $A B C-R$ value is

$$
\frac{4 \sqrt{3}+\sqrt{6}-5}{12} n+\frac{6 \sqrt{15}+6 \sqrt{2}-17 \sqrt{6}-8 \sqrt{3}+19}{12}
$$

which is uniquely attained by those trees that contain a unique vertex of degree 2 and a unique vertex of degree 3 , that is, $n_{2}=n_{3}=1$, such that each vertex of degree 2 and 3 is adjacent to only vertices of degree 4 , that is, $x_{1,2}=x_{1,3}=x_{2,3}=0, x_{2,4}=2$, and $x_{3,4}=3$. 
(3.3) For $n \geq 29$, the third maximum $A B C-R$ value is

$$
\frac{4 \sqrt{3}+\sqrt{6}-5}{12} n+\frac{18 \sqrt{15}-29 \sqrt{6}-20 \sqrt{3}+31}{12}
$$

which is uniquely attained by those trees that contain no vertex of degree 2 and exactly three vertices of degree 3 , that is, $n_{2}=0$ and $n_{3}=3$, such that each vertex of degree 3 is adjacent to three vertices of degree 4 , that is, $x_{1,3}=x_{3,3}=0$, and $x_{3,4}=9$.

\section{Maximum $A B C-R$ Index for Chemical Trees}

In this section, we present a main result which deals with the maximal chemical trees for $A B C-R$ index.

Theorem 2. Consider the set of all n-vertex chemical trees.

(1) Suppose that $n \equiv 0(\bmod 3)$.

(1.1) For $n \geq 21$, the fourth maximum $A B C-R$ value is

$$
\frac{4 \sqrt{3}+\sqrt{6}-5}{12} n+\frac{18 \sqrt{15}+36 \sqrt{2}-36 \sqrt{3}-63 \sqrt{6}+81}{36}
$$

and the equality holds if and only if $n_{2}=2$ and $n_{3}=1$ such that $x_{1,2}=x_{1,3}=x_{2,2}=x_{2,3}=0$, $x_{2,4}=4$ and $x_{3,4}=3$.

(1.2) For $n \geq 33$, the fifth maximum $A B C-R$ value is

$$
\frac{4 \sqrt{3}+\sqrt{6}-5}{12} n+\frac{54 \sqrt{15}+18 \sqrt{2}-72 \sqrt{3}-99 \sqrt{6}+117}{36},
$$

and the equality holds if and only if $n_{2}=1$ and $n_{3}=3$ such that $x_{1,2}=x_{1,3}=x_{2,3}=x_{3,3}=0$, $x_{2,4}=2$ and $x_{3,4}=9$.

(1.3) For $n \geq 33$, the sixth maximum $A B C-R$ value is

$$
\frac{4 \sqrt{3}+\sqrt{6}-5}{12} n+\frac{24 \sqrt{2}-12 \sqrt{3}-21 \sqrt{6}+33}{12}
$$

and the equality holds if and only if $n_{2}=4, n_{3}=0$ such that $x_{1,2}=x_{2,2}=0$ and $x_{2,4}=8$.

(2) Suppose that $n \equiv 1(\bmod 3)$.

(2.1) For $n \geq 37$, the fourth maximum $A B C-R$ value is

$$
\frac{4 \sqrt{3}+\sqrt{6}-5}{12} n+\frac{72 \sqrt{15}-84 \sqrt{3}-111 \sqrt{6}+123}{36}
$$

and the equality holds if and only if $n_{2}=0$ and $n_{3}=4$ such that $x_{1,3}=x_{3,3}=0$ and $x_{3,4}=12$.

(2.2) For $n \geq 37$, the fifth maximum $A B C-R$ value is

$$
\frac{4 \sqrt{3}+\sqrt{6}-5}{12} n+\frac{24 \sqrt{15}+18 \sqrt{2}-36 \sqrt{3}-66 \sqrt{6}+90}{36}
$$

and the equality holds if and only if $n_{2}=1, n_{3}=2$ such that $x_{3,3}=1, x_{1,2}=x_{1,3}=x_{2,3}=0$, $x_{2,4}=2$, and $x_{3,4}=4$.

(2.3) For $n \geq 37$, the sixth maximum $A B C-R$ value is

$$
\frac{4 \sqrt{3}+\sqrt{6}-5}{12} n+\frac{18 \sqrt{15}+54 \sqrt{2}-48 \sqrt{3}-75 \sqrt{6}+105}{36}
$$


and the equality holds if and only if $n_{2}=3$ and $n_{3}=1$ such that $x_{1,2}=x_{1,3}=x_{2,2}=x_{2,3}=0$, $x_{2,4}=6$, and $x_{3,4}=3$.

(3) Suppose that $n \equiv 2(\bmod 3)$.

(3.1) For $n \geq 29$, the fourth maximum $A B C-R$ value is

$$
\frac{4 \sqrt{3}+\sqrt{6}-5}{12} n+\frac{18 \sqrt{2}-8 \sqrt{3}-17 \sqrt{6}+25}{12},
$$

and the equality holds if and only if $n_{2}=3$ and $n_{3}=0$ such that $x_{1,2}=x_{2,2}=0$ and $x_{2,4}=6$.

For $n \geq 29$, the fifth maximum $A B C-R$ value is

$$
\frac{4 \sqrt{3}+\sqrt{6}-5}{12} n+\frac{42 \sqrt{15}-78 \sqrt{6}-48 \sqrt{3}+96}{36},
$$

and the equality holds if and only if $n_{2}=0$ and $n_{3}=3$ such that $x_{1,3}=0, x_{3,3}=1$ and $x_{3,4}=7$.

For $n \geq 29$, the sixth maximum $A B C-R$ value is

$$
\frac{4 \sqrt{3}+\sqrt{6}-5}{12} n+\frac{36 \sqrt{15}+36 \sqrt{2}-60 \sqrt{3}-87 \sqrt{6}+111}{36},
$$

and the equality holds if and only if $n_{2}=2$ and $n_{3}=2$ such that $x_{1,2}=x_{1,3}=x_{2,2}=x_{2,3}=$ $x_{3,3}=0, x_{2,4}=4$ and $x_{3,4}=6$.

Proof. First, we claim that $\theta>0.080294$ when $x_{1,2}+x_{1,3}+x_{2,2} \geq 1$ or $x_{2,3} \geq 2$. More precisely, from Equation (12),

- $\quad$ when $x_{1,2} \geq 1$,

$$
\theta \geq \frac{8 \sqrt{3}-\sqrt{6}-7}{12} \approx 0.367243>0.080294
$$

- $\quad$ when $x_{1,3} \geq 1$,

$$
\theta \geq \frac{32 \sqrt{3}-13 \sqrt{6}-19}{36} \approx 0.127285>0.080294
$$

- $\quad$ when $x_{2,2} \geq 1$,

$$
\theta \geq \frac{4 \sqrt{3}+\sqrt{6}-6 \sqrt{2}+1}{12} \approx 0.157701>0.080294
$$

- $\quad$ when $x_{2,3} \geq 2$,

$$
\theta \geq 2 \cdot \frac{8 \sqrt{3}+11 \sqrt{6}-18 \sqrt{2}-13}{36} \approx 0.130275>0.080294 .
$$

So we may assume that $x_{1,2}=x_{1,3}=x_{2,2}=0$, and $x_{2,3}=0$ or 1 . It follows from Equations (5) and (6) that

$$
x_{2,4}=2 n_{2}-x_{2,3}
$$

and

$$
2 x_{3,3}+x_{3,4}=3 n_{3}-x_{2,3}
$$

Case 1. $x_{2,3}=1$.

Observe that $n_{2} \geq 1, n_{3} \geq 1$, and thus $x_{2,4} \geq 1$ from Equation (13).

If $x_{2,4} \geq 2$, then by the Equation (12),

$$
\theta \geq \frac{8 \sqrt{3}+11 \sqrt{6}-18 \sqrt{2}-13}{36}+2 \cdot \frac{2 \sqrt{3}+2 \sqrt{6}-3 \sqrt{2}-4}{12} \approx 0.0852109>0.080294 .
$$


Suppose now that $x_{2,4}=1$. If $x_{3,3}=0$, then by Equation (14), $x_{3,4} \geq 2$, together with Equation (12), it leads to

$$
\begin{aligned}
\theta \geq & \frac{8 \sqrt{3}+11 \sqrt{6}-18 \sqrt{2}-13}{36}+\frac{2 \sqrt{3}+2 \sqrt{6}-3 \sqrt{2}-4}{12} \\
& +2 \cdot \frac{4 \sqrt{3}+4 \sqrt{6}-3 \sqrt{15}-5}{18} \\
\approx & 0.08708666>0.080294 .
\end{aligned}
$$

If $x_{3,3} \geq 1$, then by Equation (12),

$$
\begin{aligned}
\theta \geq & \frac{8 \sqrt{3}+11 \sqrt{6}-18 \sqrt{2}-13}{36}+\frac{2 \sqrt{3}+2 \sqrt{6}-3 \sqrt{2}-4}{12} \\
& +\frac{4 \sqrt{3}+7 \sqrt{6}-23}{36} \\
\approx & 0.1050251>0.080294 .
\end{aligned}
$$

Case 2. $x_{2,3}=0$.

From Equations (13) and (14), it follows that

$$
x_{2,4}=2 n_{2}
$$

and

$$
2 x_{3,3}+x_{3,4}=3 n_{3} .
$$

If $x_{3,3} \geq 3$, then by Equation (12),

$$
\theta \geq 3 \cdot \frac{4 \sqrt{3}+7 \sqrt{6}-23}{36} \approx 0.0895526>0.0802936 .
$$

If $x_{3,3}=2$, then $n_{3} \geq 3$, and $x_{3,4} \geq 5$ from Equation (14), and thus by Equation (12),

$$
\theta \geq 2 \cdot \frac{4 \sqrt{3}+7 \sqrt{6}-23}{36}+5 \cdot \frac{4 \sqrt{3}+4 \sqrt{6}-3 \sqrt{15}-5}{18} \approx 0.0894829>0.0802936 .
$$

Now, we consider the two cases: $x_{3,3}=1$ and $x_{3,3}=0$.

Subcase 2.1. $x_{3,3}=1$.

Clearly, $n_{3} \geq 2$. The proofs will be partitioned into several parts according to the value of $n_{3}$ : $n_{3}=2, n_{3}=3, n_{3} \geq 4$.

Firstly suppose that $n_{3}=2$, then, $x_{3,4}=4$ from Equation (14). Note that the case $n_{2}=0$ is known to belong to one of the first three minimum $\theta$ values, see Theorem 1-(1.3). If $n_{2}=1$, then $n \equiv 1(\bmod 3)$ from Equation (8), $x_{2,4}=2$ from Equation (17), and by Equation (12),

$$
\begin{aligned}
\theta & =2 \cdot \frac{2 \sqrt{3}+2 \sqrt{6}-3 \sqrt{2}-4}{12}+\frac{4 \sqrt{3}+7 \sqrt{6}-23}{36}+4 \cdot \frac{4 \sqrt{3}+4 \sqrt{6}-3 \sqrt{15}-5}{18} \\
& \approx 0.0737492 .
\end{aligned}
$$

If $n_{2} \geq 2$, then, $x_{2,4} \geq 4$ from Equation (17), and by Equation (12),

$$
\begin{aligned}
\theta & \geq 4 \cdot \frac{2 \sqrt{3}+2 \sqrt{6}-3 \sqrt{2}-4}{12}+\frac{4 \sqrt{3}+7 \sqrt{6}-23}{36}+4 \cdot \frac{4 \sqrt{3}+4 \sqrt{6}-3 \sqrt{15}-5}{18} \\
& \approx 0.09382262>0.0802936
\end{aligned}
$$


Next, suppose that $n_{3}=3$, then $x_{3,4}=7$ from Equation (14). If $n_{2}=0$, then $n \equiv 2(\bmod 3)$ from Equation (8), $x_{2,4}=0$ from Equation (17), and by Equation (12),

$$
\theta=\frac{4 \sqrt{3}+7 \sqrt{6}-23}{36}+7 \cdot \frac{4 \sqrt{3}+4 \sqrt{6}-3 \sqrt{15}-5}{18} \approx 0.0715445
$$

If $n_{2} \geq 1$, then $x_{2,4} \geq 2$ from Equation (17), and by Equation (12),

$$
\begin{aligned}
\theta & \geq 2 \cdot \frac{2 \sqrt{3}+2 \sqrt{6}-3 \sqrt{2}-4}{12}+\frac{4 \sqrt{3}+7 \sqrt{6}-23}{36}+7 \cdot \frac{4 \sqrt{3}+4 \sqrt{6}-3 \sqrt{15}-5}{18} \\
& \approx 0.0916179>0.0802936
\end{aligned}
$$

Finally, if $n_{3} \geq 4$, then $x_{3,4} \geq 10$ from Equation (16), and by Equation (12),

$$
\begin{aligned}
\theta & \geq \frac{4 \sqrt{3}+7 \sqrt{6}-23}{36}+10 \cdot \frac{4 \sqrt{3}+4 \sqrt{6}-3 \sqrt{15}-5}{18} \\
& \approx 0.0894132>0.0802936
\end{aligned}
$$

Subcase 2.2. $x_{3,3}=0$.

In this case, $x_{3,4}=3 n_{3}$ from Equation (18). This time, we partition the proofs according to the value of $n_{2}: n_{2}=0, n_{2}=1, n_{2}=2, n_{2}=3, n_{2}=4, n_{2} \geq 5$.

Firstly suppose that $n_{2}=0$, that is, $x_{2,4}=0$ from Equation (17). Note that the cases $n_{3}=0,1,2,3$ were known to belong to the first three minimum $\theta$ value, see Theorem 1 . If $n_{3}=4$, then $n \equiv 1(\bmod 3)$ from Equation (8), $x_{3,4}=12$, and by Equation (12),

$$
\theta=12 \cdot \frac{4 \sqrt{3}+4 \sqrt{6}-3 \sqrt{15}-5}{18} \approx 0.0714748
$$

If $n_{3} \geq 5$, then $x_{3,4} \geq 15$, and by Equation (12),

$$
\theta \geq 15 \cdot \frac{4 \sqrt{3}+4 \sqrt{6}-3 \sqrt{15}-5}{18} \approx 0.08934345>0.0802936 .
$$

Next, suppose that $n_{2}=1$, that is, $x_{2,4}=2$ from Equation (17). Note that the cases $n_{3}=0,1,2$ were known to belong to the first three minimum $\theta$ values, see Theorem 1 . If $n_{3}=3$, then $n \equiv 0(\bmod 3)$ from Equation (8), $x_{3,4}=9$, and by Equation (12),

$$
\theta=2 \cdot \frac{2 \sqrt{3}+2 \sqrt{6}-3 \sqrt{2}-4}{12}+9 \cdot \frac{4 \sqrt{3}+4 \sqrt{6}-3 \sqrt{15}-5}{18} \approx 0.0736795
$$

If $n_{3} \geq 4$, then $x_{3,4} \geq 12$, and by Equation (12),

$$
\begin{aligned}
\theta & \geq 2 \cdot \frac{2 \sqrt{3}+2 \sqrt{6}-3 \sqrt{2}-4}{12}+12 \cdot \frac{4 \sqrt{3}+4 \sqrt{6}-3 \sqrt{15}-5}{18} \\
& \approx 0.091548>0.0802936
\end{aligned}
$$

Now, suppose that $n_{2}=2$, that is, $x_{2,4}=4$ from Equation (17). The case $n_{3}=0$ was known to belong to one of the first three minimum $\theta$ values, see Theorem 1-(2.2). If $n_{3}=1$, then $n \equiv 0(\bmod 3)$ from Equation (8), $x_{3,4}=3$, and by Equation (12),

$$
\theta=4 \cdot \frac{2 \sqrt{3}+2 \sqrt{6}-3 \sqrt{2}-4}{12}+3 \cdot \frac{4 \sqrt{3}+4 \sqrt{6}-3 \sqrt{15}-5}{18} \approx 0.0580155
$$


If $n_{3}=2$, then $n \equiv 2(\bmod 3)$ from Equation $(8), x_{3,4}=6$, and by Equation (12),

$$
\theta=4 \cdot \frac{2 \sqrt{3}+2 \sqrt{6}-3 \sqrt{2}-4}{12}+6 \cdot \frac{4 \sqrt{3}+4 \sqrt{6}-3 \sqrt{15}-5}{18} \approx 0.07588419 .
$$

If $n_{3} \geq 3$, then $x_{3,4} \geq 9$, and by Equation (12),

$$
\begin{aligned}
\theta & \geq 4 \cdot \frac{2 \sqrt{3}+2 \sqrt{6}-3 \sqrt{2}-4}{12}+9 \cdot \frac{4 \sqrt{3}+4 \sqrt{6}-3 \sqrt{15}-5}{18} \\
& \approx 0.09375289>0.0802936
\end{aligned}
$$

Suppose that $n_{2}=3$, that is, $x_{2,4}=6$ from Equation (17). If $n_{3}=0$, then $n \equiv 2(\bmod 3)$ from Equation (8), $x_{3,4}=0$, and by Equation (12),

$$
\theta=6 \cdot \frac{2 \sqrt{3}+2 \sqrt{6}-3 \sqrt{2}-4}{12} \approx 0.0602202
$$

If $n_{3}=1$, then $n \equiv 1(\bmod 3)$ from Equation $(8), x_{3,4}=3$, and by Equation (12),

$$
\theta=6 \cdot \frac{2 \sqrt{3}+2 \sqrt{6}-3 \sqrt{2}-4}{12}+3 \cdot \frac{4 \sqrt{3}+4 \sqrt{6}-3 \sqrt{15}-5}{18} \approx 0.0780889
$$

If $n_{3} \geq 2$, then $x_{3,4} \geq 6$, and by Equation (12),

$$
\begin{aligned}
\theta & \geq 6 \cdot \frac{2 \sqrt{3}+2 \sqrt{6}-3 \sqrt{2}-4}{12}+6 \cdot \frac{4 \sqrt{3}+4 \sqrt{6}-3 \sqrt{15}-5}{18} \\
& \approx 0.0959576>0.0802936
\end{aligned}
$$

Suppose that $n_{2}=4$, that is, $x_{2,4}=8$ from Equation (17). If $n_{3}=0$, then $n \equiv 0(\bmod 3)$ from Equation (8), $x_{3,4}=0$, and by Equation (12),

$$
\theta=8 \cdot \frac{2 \sqrt{3}+2 \sqrt{6}-3 \sqrt{2}-4}{12} \approx 0.0802936
$$

If $n_{3} \geq 1$, then $x_{3,4} \geq 3$, and by Equation (12),

$$
\begin{aligned}
\theta & \geq 8 \cdot \frac{2 \sqrt{3}+2 \sqrt{6}-3 \sqrt{2}-4}{12}+3 \cdot \frac{4 \sqrt{3}+4 \sqrt{6}-3 \sqrt{15}-5}{18} \\
& \approx 0.0981623>0.0802936
\end{aligned}
$$

Finally, if $n_{2} \geq 5$, then $x_{2,4} \geq 10$ from Equation (17), and by Equation (12),

$$
\theta \geq 10 \cdot \frac{2 \sqrt{3}+2 \sqrt{6}-3 \sqrt{2}-4}{12} \approx 0.100367>0.0802936 .
$$

In conclusion, we obtain the following

(i) If $n \equiv 0(\bmod 3)$, then the fourth, fifth and sixth minimum $\theta$ values are $0.0580155,0.0736795$ and 0.0802936 , respectively.

(ii) If $n \equiv 1(\bmod 3)$, then the fourth, fifth and sixth minimum $\theta$ values are $0.0714748,0.0737492$ and 0.0780889 , respectively.

(iii) If $n \equiv 2(\bmod 3)$, then the fourth, fifth and sixth minimum $\theta$ values are $0.0602202,0.0715445$ and 0.07588419 , respectively.

Now, the Equation (11) implies the fourth, fifth and sixth maximum $A B C-R$.

In Figures 1-3, the chemical trees with the smallest numbers of vertices in Theorem 2 are listed. 


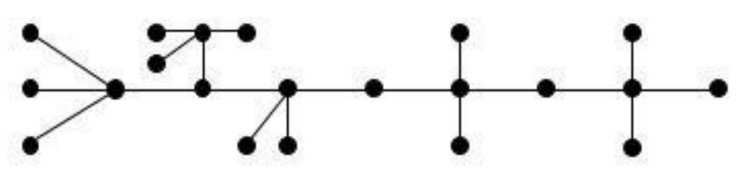

(A)

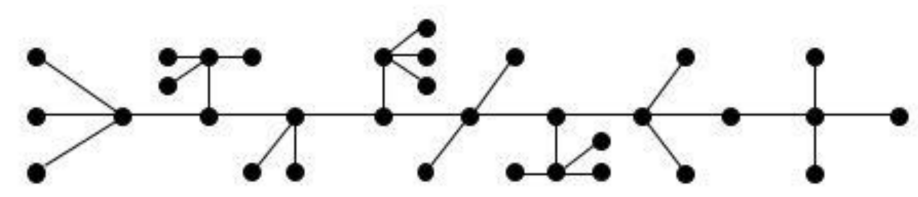

(B)

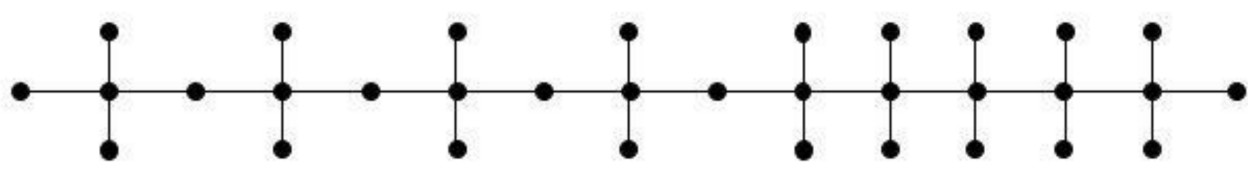

(C)

Figure 1. Chemical trees with the fourth (A), the fifth (B) and the sixth (C) maximum $A B C-R$ values in Theorem 2-(1).

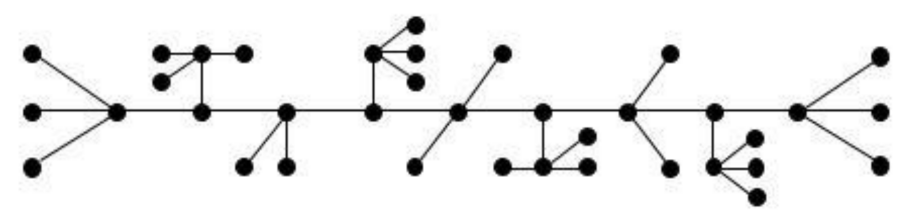

(D)

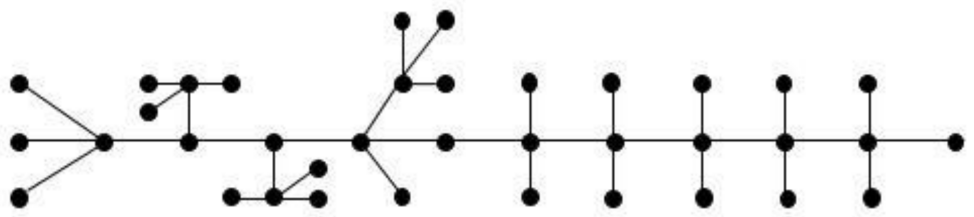

(E)

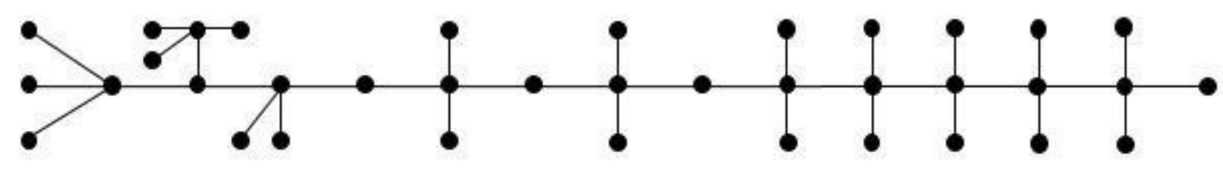

(F)

Figure 2. Chemical trees with the fourth (D), the fifth (E) and the sixth (F) maximum $A B C-R$ values in Theorem 2-(2). 


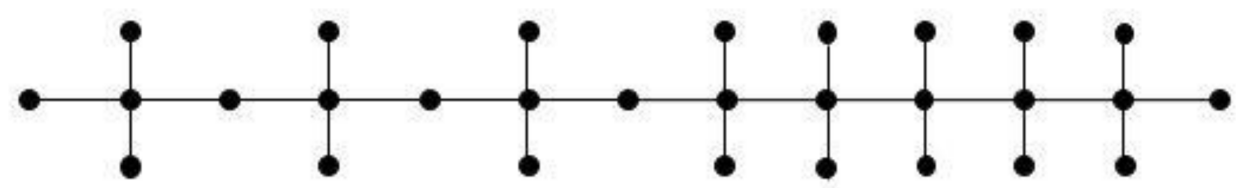

(G)

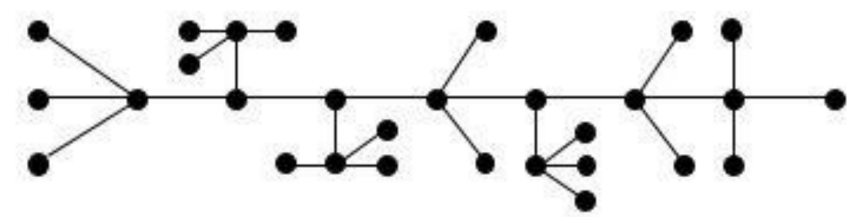

(H)

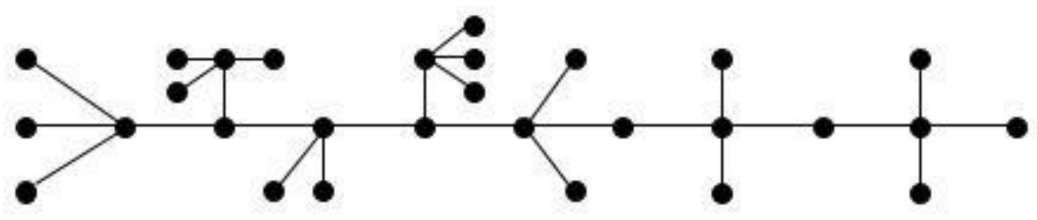

(I)

Figure 3. Chemical trees with the fourth $(\mathrm{G})$, the fifth $(\mathrm{H})$ and the sixth (I) maximum $A B C-R$ values in Theorem 2-(3).

\section{Upper Bound for $A B C-R$ Index of Molecular Trees}

In this section, we consider the class of molecular tress and investigated the sharp bound on $A B C-R$ for this class of graphs.

Let $\mathcal{T}_{n, n_{1}}$ be the set of molecular trees satisfying

$$
\begin{gathered}
x_{1,4}=n_{1}, \\
x_{2,2}=n-2 n_{1}+3-\frac{1}{3} x_{2,3}
\end{gathered}
$$

and

$$
x_{2,4}=n_{1}-4-\frac{2}{3} x_{2,3}
$$

Theorem 3 ([19]). Let $T$ be a molecular tree with $n$ vertices, $n_{1} \geq 5$ of which are pendant vertices. Then

$$
(A B C)(T) \leq \frac{\sqrt{2}}{2} n+\frac{\sqrt{3}-\sqrt{2}}{2} n_{1}-\frac{\sqrt{2}}{2}
$$

with equality holds if and only if $T \in \mathcal{T}_{n, n_{1}}$.

Obviously, from Equation (1) we obtain

$$
\begin{aligned}
(A B C-R)(T)= & \frac{\sqrt{2}-1}{\sqrt{3}} x_{1,3}+\frac{\sqrt{3}-1}{2} x_{1,4}+\frac{\sqrt{2}-1}{2} x_{2,2}+\frac{\sqrt{3}-1}{\sqrt{6}} x_{2,3}+ \\
& \frac{1}{\sqrt{8}} x_{2,4}+\frac{1}{3} x_{3,3}+\frac{\sqrt{5}-1}{\sqrt{12}} x_{3,4}+\frac{\sqrt{6}-1}{4} x_{4,4}
\end{aligned}
$$


Now let $\mathcal{T}_{n, n_{1}}^{\prime}$ be the set of molecular trees satisfying

$$
\begin{gathered}
x_{1,4}=n_{1}, \\
x_{2,2}=n-2 n_{1}+3,
\end{gathered}
$$

and

$$
x_{2,4}=n_{1}-4
$$

Theorem 4. Let $T$ be a molecular tree of order $n$ and $n_{1} \geq 5$ pendant vertices, then

$$
(A B C-R)(T) \leq \frac{\sqrt{2}-1}{2} n+\frac{2-3 \sqrt{2}+2 \sqrt{3}}{4} n_{1}+\frac{1}{\sqrt{2}}-\frac{3}{2}
$$

with equality holds if and only if $T \in \mathcal{T}_{n, n_{1}}^{\prime}$.

Proof. Since $T$ is a molecular tree, we have Equations (2)-(7). Suppose that

$$
\begin{aligned}
& f_{1}=x_{1,2}+x_{1,3}+x_{1,4} \\
& f_{2}=x_{1,2}+x_{2,3} \\
& f_{3}=x_{1,3}+x_{2,3}+2 x_{3,3}+x_{3,4} \\
& f_{4}=x_{1,4}+x_{3,4}+2 x_{4,4}
\end{aligned}
$$

that is,

$$
\begin{aligned}
& f_{1}=n_{1} \\
& f_{2}=2 n_{2}-2 x_{2,2}-x_{2,4} \\
& f_{3}=3 n_{3} \\
& f_{4}=4 n_{4}-x_{2,4}
\end{aligned}
$$

we have

$$
\begin{aligned}
\sum_{i=1}^{4} f_{i} & =2(n-1)-2\left(x_{2,2}+x_{2,4}\right) \\
\sum_{i=1}^{4} \frac{1}{i} f_{i} & =n-\left(x_{2,2}+\frac{3}{4} x_{2,4}\right)
\end{aligned}
$$

implying that

$$
\begin{aligned}
& x_{2,2}=\frac{3}{2} \sum_{i=1}^{4} f_{i}-4 \sum_{i=1}^{4} \frac{1}{i} f_{i}+n+3 \\
& x_{2,4}=-2 \sum_{i=1}^{4} f_{i}+4 \sum_{i=1}^{4} \frac{1}{i} f_{i}-4 .
\end{aligned}
$$

Thus we have

$$
\begin{aligned}
& x_{1,4}=n_{1}-x_{1,2}-x_{1,3} \\
& x_{2,2}=n-2 n_{1}+3-x_{1,2}-\frac{1}{3} x_{1,3}-\frac{1}{3} x_{2,3}+\frac{1}{3} x_{3,3}+\frac{2}{3} x_{3,4}+x_{4,4} \\
& x_{2,4}=n_{1}-4+x_{1,2}+\frac{1}{3} x_{1,3}-\frac{2}{3} x_{2,3}-\frac{4}{3} x_{3,3}-\frac{5}{3} x_{3,4}-2 x_{4,4} .
\end{aligned}
$$


Substituting them back into Equation (19), we have

$$
\begin{aligned}
(A B C-R)(T)= & \frac{\sqrt{2}-1}{2} n+\frac{2-3 \sqrt{2}+2 \sqrt{3}}{4} n_{1}+\frac{1}{\sqrt{2}}-\frac{3}{2} \\
& +\frac{4-\sqrt{2}-2 \sqrt{3}}{4} x_{1,2}+\frac{8-\sqrt{2}-10 \sqrt{3}+4 \sqrt{6}}{12} x_{1,3} \\
& +\frac{1+\sqrt{2}-\sqrt{6}}{6} x_{2,3}+\frac{1-\sqrt{2}}{6} x_{3,3} \\
& +\frac{2 \sqrt{15}-4-\sqrt{2}-2 \sqrt{3}}{12} x_{3,4}+\frac{\sqrt{6}-3}{4} x_{4,4} \\
\approx & \frac{\sqrt{2}-1}{2} n+\frac{2-3 \sqrt{2}+2 \sqrt{3}}{4} n_{1}+\frac{1}{\sqrt{2}}-\frac{3}{2} \\
& -0.219579 x_{1,2}-0.078064 x_{1,3}-0.005879 x_{2,3} \\
& -0.069036 x_{3,3}-0.094362 x_{3,4}-0.137628 x_{4,4}
\end{aligned}
$$

with negative coefficients $x_{1,2}, x_{1,3}, x_{2,3}, x_{3,3}, x_{3,4}$ and $x_{4,4}$. Thus

$$
(A B C-R)(T) \leq \frac{\sqrt{2}-1}{2} n+\frac{2-3 \sqrt{2}+2 \sqrt{3}}{4} n_{1}+\frac{1}{\sqrt{2}}-\frac{3}{2}
$$

and equality in above holds if and only if $x_{1,2}=x_{1,3}=x_{2,3}=x_{3,3}=x_{3,4}=x_{4,4}=0$, or equivalently, $x_{1,4}=n_{1}, x_{2,2}=n-2 n_{1}+3, x_{2,4}=n_{1}-4$, i.e., $T \in \mathcal{T}_{n, n_{1}}^{\prime}$.

\section{Conclusions}

In this paper, we considered more maximum values of the difference $A B C-R$, where $A B C$ and $R$ are the atom-bond connectivity index and Randić index, respectively. In particular, we characterized the fourth, the fifth and the sixth maximum chemical trees with respect to the invariant $A B C-R$, and thus extended the result by Ali and $\mathrm{Du}$ [24] in 2017. It is very challenging to find more maximum values of $A B C-R$ invariant unless new efficient method is introduced. By using the technique from [19], we also obtained a sharp upper bound for the $A B C-R$ index of molecular (or chemical) trees with fixed number of pendant vertices. The work on bounds for the $A B C-R$ index of general graphs and trees is widely open and one can consider many directions.

Author Contributions: Conceptualization, R.H. and Z.D.; methodology, W.N.N.N.W.Z.; validation, R.H., Z.D. and M.K.J.; formal analysis, W.N.N.N.W.Z.; investigation, W.N.N.N.W.Z., R.H. and Z.D.; resources, R.H.; writing-original draft preparation, R.H.; writing—review and editing, R.H., Z.D. and M.K.J.; supervision, R.H. and Z.D.; project administration, R.H.; funding acquisition, R.H. All authors have read and agreed to the published version of the manuscript

Funding: This research received no external funding.

Acknowledgments: This research is supported by the Research Intensified Grant Scheme (RIGS), Phase 1/2019, Universiti Malaysia Terengganu, Malaysia with Grant Vot. 55192/6. The authors would like to thanks the referees for the constructive and valuable comments that improved the paper.

Conflicts of Interest: The authors declare no conflict of interest.

\section{References}

1. West, D.B. Introduction to Graph Theory, 2nd ed.; Prentice Hall, Inc.: Upper Saddle River, NJ, USA, 2001.

2. Devillers, J.; Balaban, A.T. (Eds.) Topological Indices and Related Descriptors in QSAR and QSPR; Gordon and Breach: Amsterdam, The Netherlands, 1999.

3. Todeschini, R.; Consonni, V. Handbook of Molecular Descriptors; Wiley-VCH: Weinheim, Germany, 2000.

4. Randić, M. On characterization of molecular branching. J. Am. Chem. Soc. 1975, 97, 6609-6615. [CrossRef]

5. Gutman, I.; Furtula, B. Recent results in the theory of Randić index. In Mathematical Chemistry Monograph. 6; University of Kragujevac: Kragujevac, Serbia, 2008. 
6. Li, X.; Gutman, I. Mathematical aspects of Randić-type molecular structure descriptors. In Mathematical Chemistry Monograph. 1; University of Kragujevac: Kragujevac, Serbia, 2006.

7. Li, X.; Shi, Y. A survey on the Randić index. MATCH Commun. Math. Comput. Chem. 2008, 59, 127-156.

8. Randić, M. On the history of the Randić index and emerging hostility towards chemical graph theory. MATCH Commun. Math. Comput. Chem. 2008, 59, 5-124.

9. Husin, N.M.; Hasni, R.; Du, Z.; Ali, A. More results on extremum Randić indices of (molecular) trees. Filomat 2018, 32, 3581-3590. [CrossRef]

10. Li, J.; Balachandran, S.; Ayyaswamy, S.K.; Venkatakrishnan, Y.B. The Randić indices of trees, unicyclic graphs and bicyclic graphs. Ars Combin. 2016, 127, 409-419.

11. Estrada, E.; Torres, L.; Rodríguez, L.; Gutman, I. An atom-bond connectivity index: Modelling the enthalpy of formation of alkanes. Indian J. Chem. Sect. A 1998, 37, 849-855.

12. Estrada, E. Atom-bond connectivity and energetic of branched alkanes. Chem. Phys. Lett. 2008, 463, $422-425$. [CrossRef]

13. Gutman, I.; Furtula, B.; Ahmadi, M.B.; Hosseini, S.A.; Nowbandegani, P.S.; Zarrinderakht, M. The $A B C$ index conundrum. Filomat 2013, 27, 1075-1083. [CrossRef]

14. Cui, Q.; Qian, Q.; Zhong, L. The maximum atom-bond connectivity index for graphs with edge-connectivity one. Discrete Appl. Math. 2017, 220, 170-173. [CrossRef]

15. Dimitrov, D. On the structural properties of trees with minimal atom-bond connectivity index III: Bounds on $B_{1}$ - and $B_{2}$-branches. Discrete Appl. Math. 2016, 204, 90-116. [CrossRef]

16. Gao, Y.; Shao, Y. The smallest $A B C$ index of trees with $n$ pendant vertices. MATCH Commun. Math. Comput. Chem. 2016, 76, 141-158.

17. Shao, Z.; Wu, P.; Gao, Y.; Gutman, I.; Zhang, Z. On the maximum $A B C$ index of graphs without pendent vertices. Discrete Appl. Math. 2017, 315, 298-312. [CrossRef]

18. Xing, R.; Zhou, B.; Dong, F. On the atom-bond connectivity index of connected graphs. Discrete Appl. Math. 2011, 159, 1617-1630. [CrossRef]

19. Xing, R.; Zhou, B.; Du, Z. Further results on atom-bond connectivity index of trees. Discrete Appl. Math. 2010, 158, 1536-1545. [CrossRef]

20. Das, K.C.; Das, S.; Zhou, B. Sum-connectivity index. Front. Math. China 2016, 11, 47-54. [CrossRef]

21. Das, K.C.; Trinajstić, N. Comparison between the first geometric-arithmetic index and atom-bond connectivity index. Chem. Phys. Lett. 2010, 497, 149-151. [CrossRef]

22. Raza, Z.; Bhatti, A.A.; Ali, A. More comparison between the first geometric-arithmetic index and atom-bond connectivity index. Miskolc Math. Notes 2016, 17, 561-570. [CrossRef]

23. Zhong, L.; Cui, Q. On a relation between the atom-bond connectivity and the first geometric-arithmetic indices. Discrete Appl. Maths 2015, 185, 249-253. [CrossRef]

24. Ali, A.; Du, Z. On the difference between atom-bond connectivity index and Randić index of binary and chemical trees. Int. J. Quantum Chem. 2017, 117, e25446. [CrossRef]

25. Riaz, A.; Ellahi, R.; Bhatti, M.M.; Marin, M. Study of heat and mass transfer in the Erying-Powell model of fluid propagating peristaltically through a rectangular compliant channel. Heat Transfer Res. 2019, 50, 1539-1560. [CrossRef]

26. Gutman, I.; Miljkovic, O.; Caporossi, G.; Hansen, P. Alkanes with small and large Randić connectivity indices. Chem. Phys. Lett. 1999, 306, 366-372. [CrossRef]

(C) 2020 by the authors. Licensee MDPI, Basel, Switzerland. This article is an open access article distributed under the terms and conditions of the Creative Commons Attribution (CC BY) license (http://creativecommons.org/licenses/by/4.0/). 